\title{
A Population Physiologically-Based Pharmacokinetic Model to Characterize Antibody Disposition in Pediatrics and Evaluation of the Model using Infliximab
}

\author{
Hsuan Ping Chang ${ }^{1}$, Valentina Shakhnovich ${ }^{2}$, Adam Frymoyer ${ }^{3}$, Ryan Funk ${ }^{4}$, Mara \\ Becker $^{4}$, K.T. Park ${ }^{4}$, and Dhaval Shah ${ }^{1}$ \\ ${ }^{1}$ University at Buffalo - The State University of New York \\ ${ }^{2}$ Children's Mercy Kansas City \\ ${ }^{3}$ Stanford Univ \\ ${ }^{4}$ Affiliation not available
}

February 24, 2021

\begin{abstract}
Aims: In order to better predict the pharmacokinetics (PK) of antibodies in children, and to facilitate dose optimization of antibodies in pediatric patients, there is a need to develop systems PK models that integrate ontogeny related changes in human physiological parameters. Methods: A population-based physiological-based PK (PBPK) model to characterize antibody PK in pediatrics has been developed, by incorporating age-related changes in body weight, organ weight, organ blood flow rate, and interstitial volumes in a previously published platform model. The model was further used to perform Monte Carlo simulations to investigate clearance vs. age and dose-exposure relationship for infliximab. Results: By estimating only one parameter and associated interindividual variability, the model was able to characterize clinical PK of infliximab from two pediatric cohorts ( $\mathrm{n}=141,4-19$ years) reasonably well. Model simulations demonstrated that only $50 \%$ of children reached desired trough concentrations when receiving FDA-labelled dosing regimen for infliximab, suggesting that higher doses and/or more frequent dosing are needed to achieve target trough concentrations of this antibody. Conclusion: The pediatric PBPK model presented here can serve as a framework to characterize the PK of antibodies in pediatric patients. The model can also be applied to other protein therapeutics to advance precision medicine paradigm and optimize antibody dosing regimens in children.
\end{abstract}

\section{Hosted file}

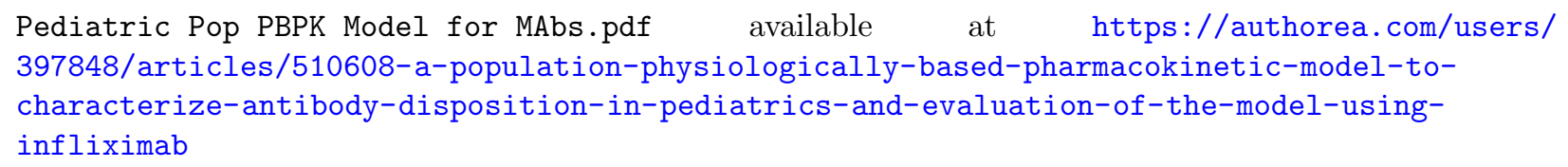




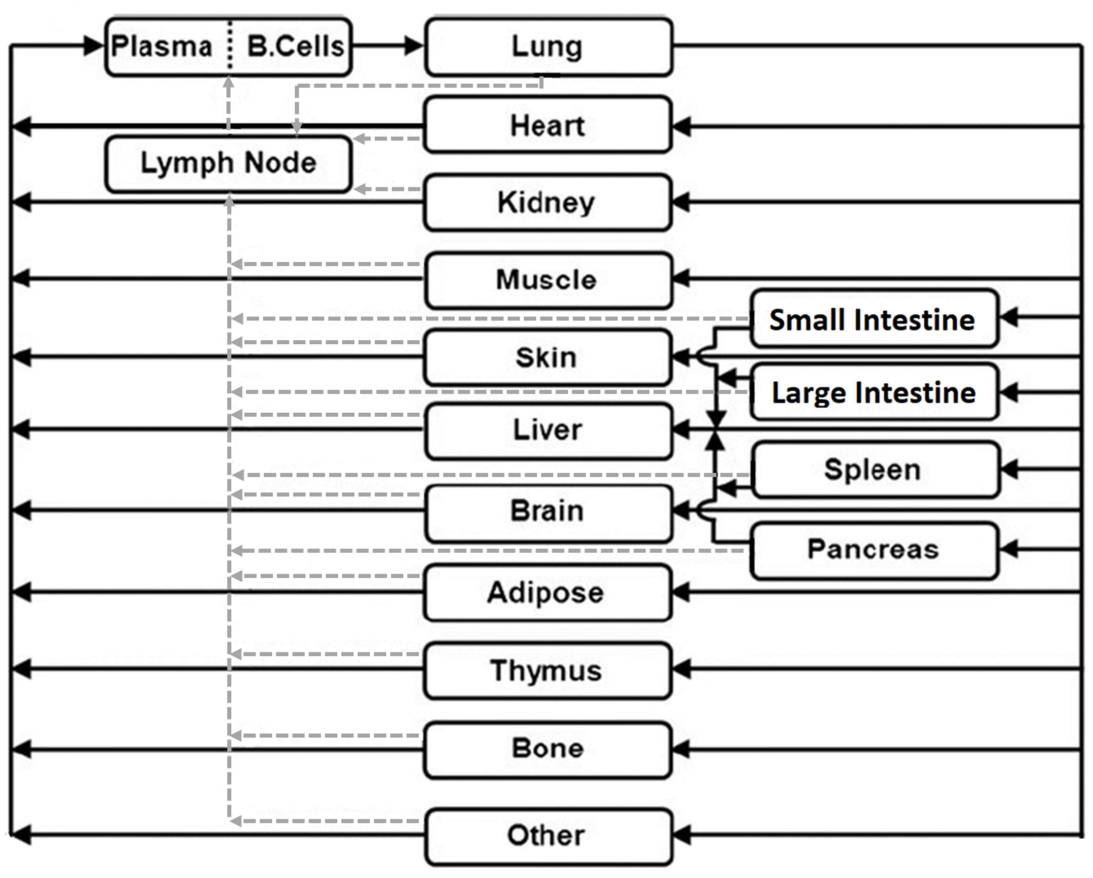

$\longleftarrow$ Blood flow rate $\left(Q_{i}\right)=f($ age,$B W$, sex $)$

Organ Organ weight $=f($ age,$B W$, sex $)$

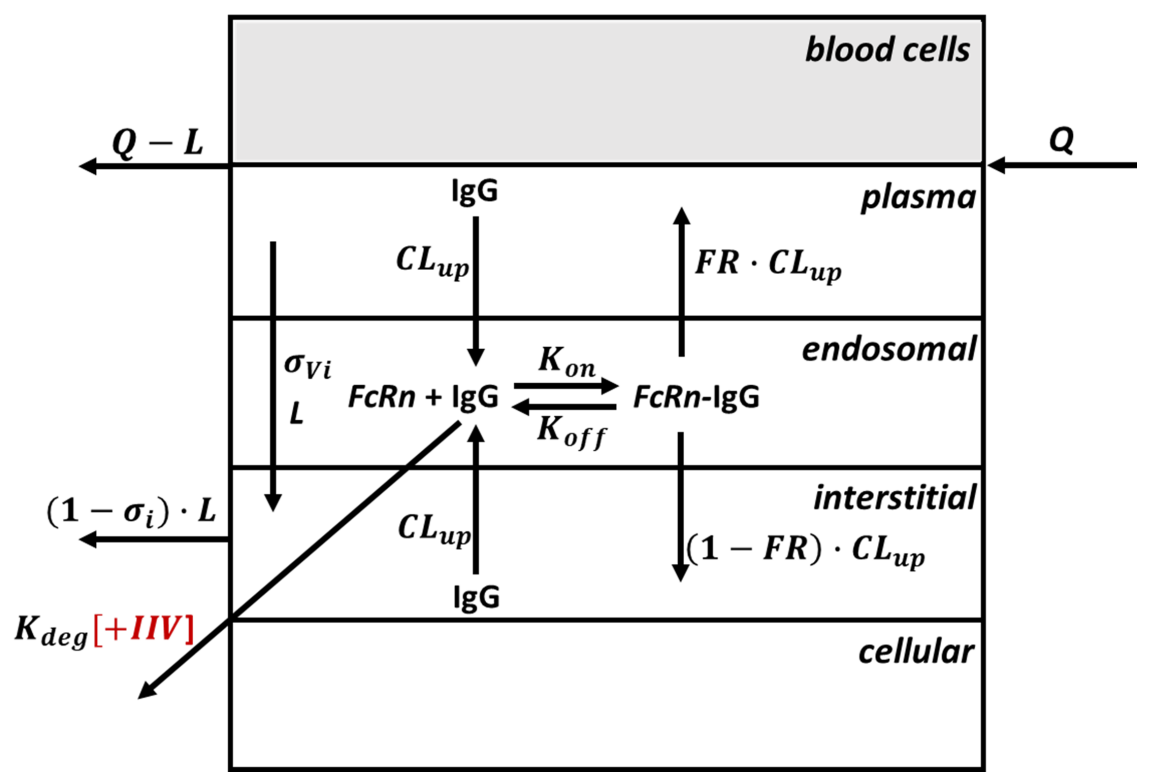



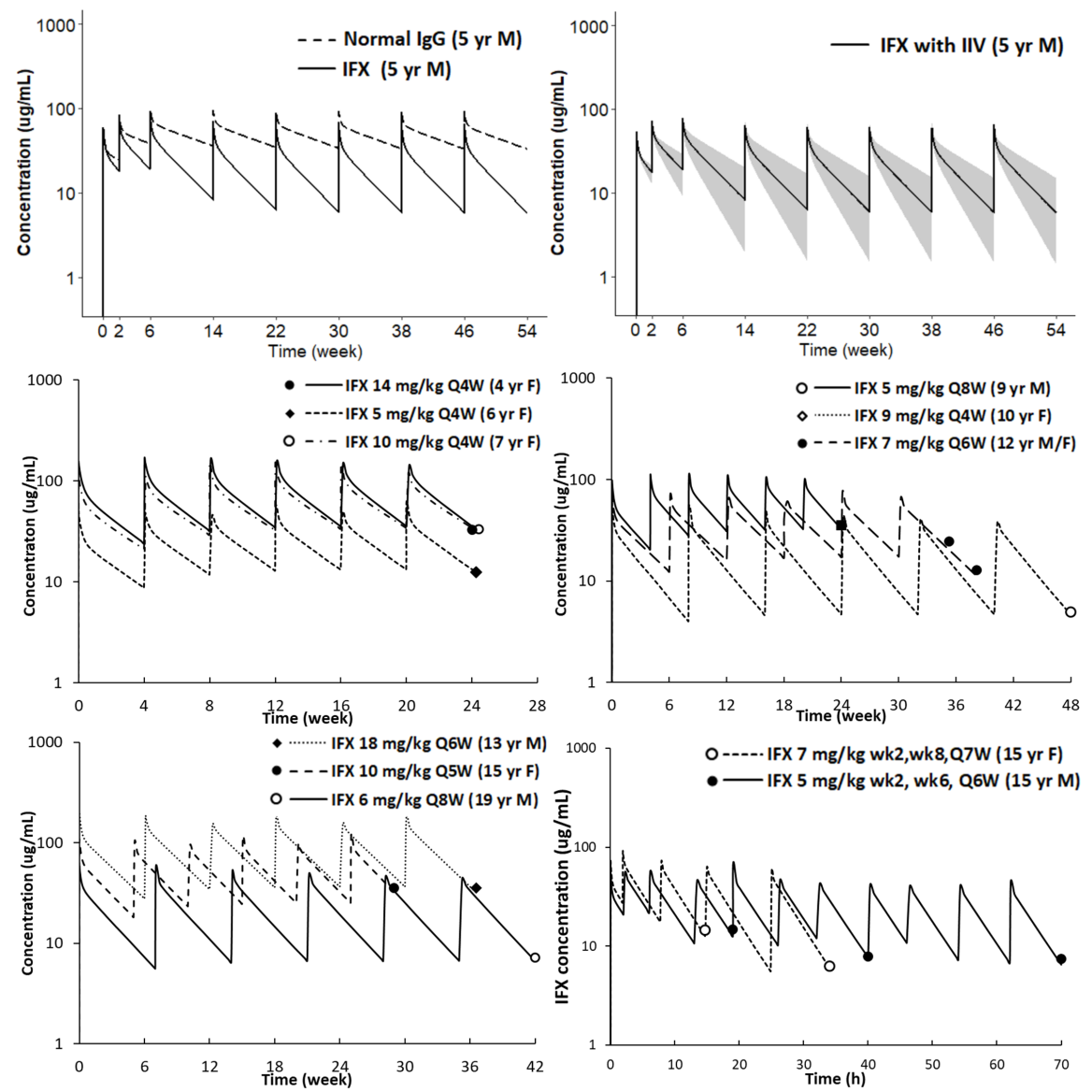

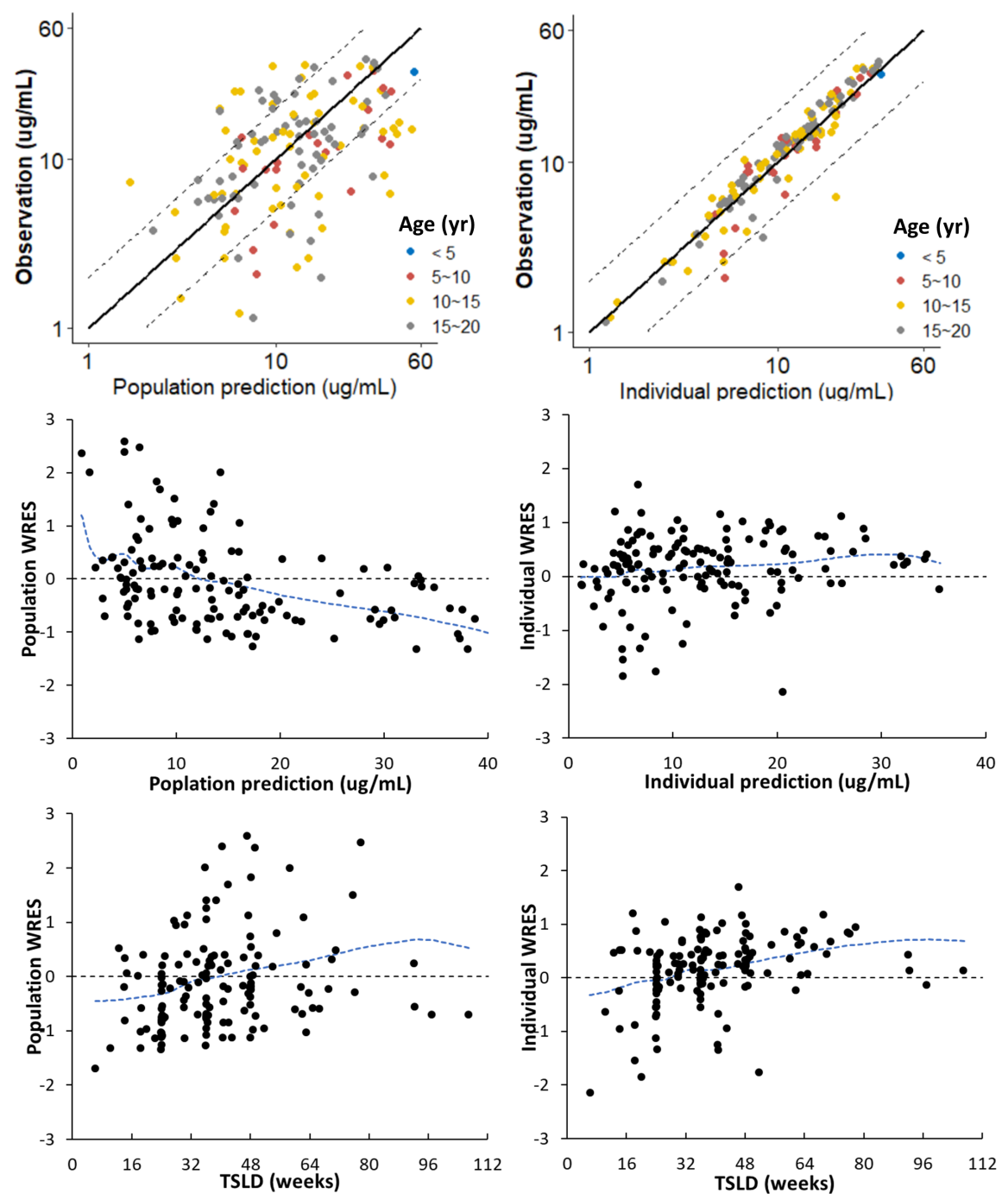

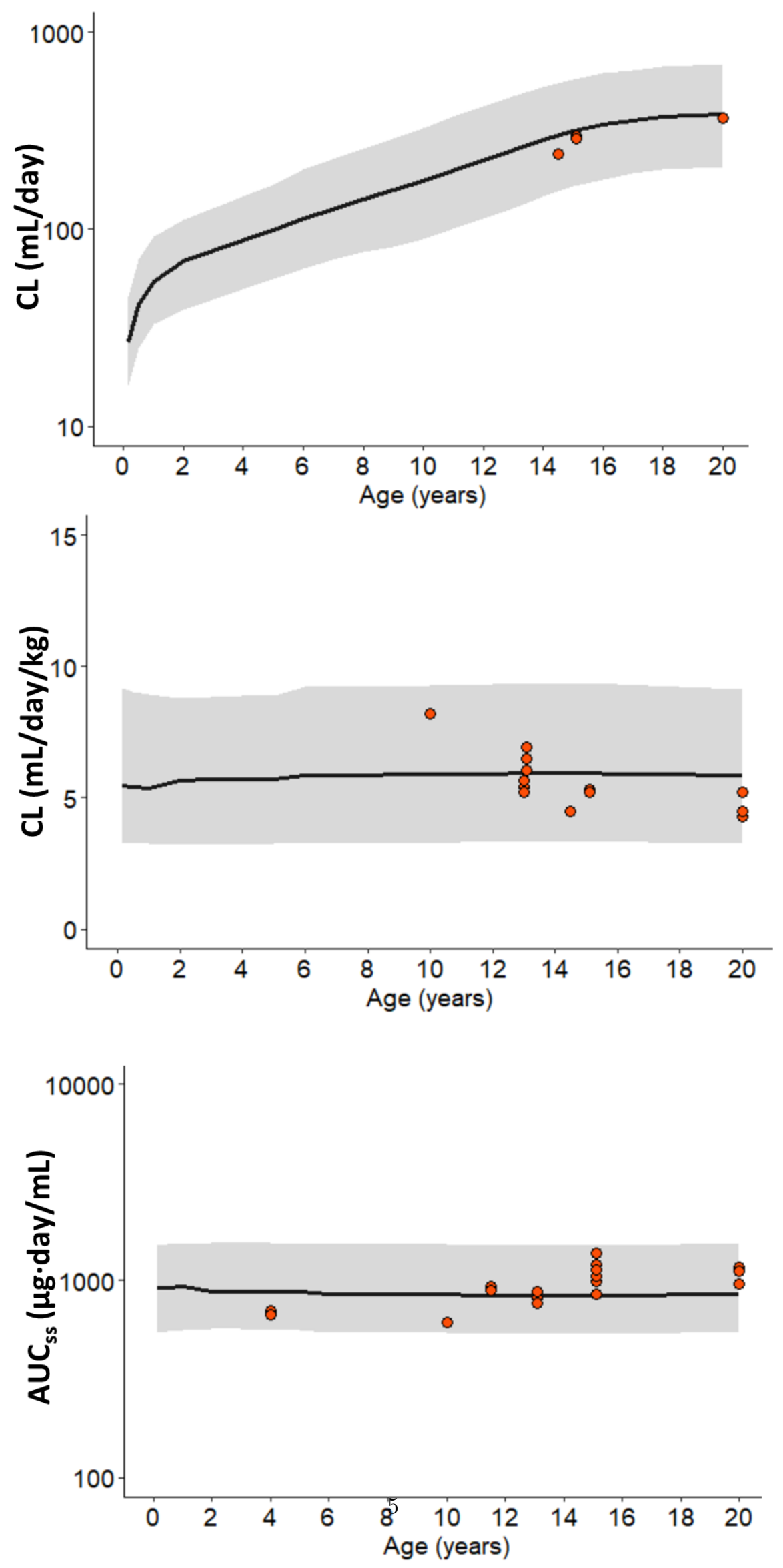